Check for updates

Cite this: RSC Adv., 2018, 8, 26212

Received 29th May 2018

Accepted 3rd July 2018

DOI: $10.1039 / \mathrm{c} 8 \mathrm{ra04581j}$

rsc.li/rsc-advances

\section{Fluorescence enhancement of carbon dots by graphene for highly sensitive detection of tetracycline hydrochloride $\uparrow$}

\author{
Wei He, (D) Xiangying Sun, ${ }^{*}$ Wenting Weng (D) and Bin Liu
}

In this study, fluorescence of self-assembled multilayers (SAMs) of carbon dots (CDs) was found to be enhanced by graphene. The number of polyelectrolyte layers can be tuned to control the distance between CDs and graphene in SAMs. The enhanced fluorescence efficiency was found to be dependent on graphene concentration, degree of graphene reduction, and the distance between CDs and graphene surface. When graphene concentration and polyelectrolyte bilayer number were set to $0.005 \mathrm{mg} \mathrm{mL}^{-1}$ and 3 layers, respectively, fluorescence intensity of CDs could be increased up to 3.2 times. Tetracycline hydrochloride (Tc) could be detected by the established SAMs in the presence of graphene as the sensitivity was 2 orders higher than that of SAMs in the absence of graphene. Limit of detection of this sensing system was $0.9284 \mathrm{nM}$, which is $1-3$ orders of magnitude lower than those of most of reported fluorescence sensors. This method could be successfully applied to detect trace Tc in milk samples.

\section{Introduction}

Tetracycline hydrochloride (Tc) is a well-known antibacterial drug, widely used in pharmaceuticals, food and environmental fields. ${ }^{1,2}$ Although Tc is beneficial for treatment of some diseases, its residues in food may lead to some allergic reactions. According to European Union law, the maximum amount of tetracycline hydrochloride in milk must not exceed $100 \mathrm{ng}$ $\mathrm{mL}^{-1}$ (ca. $\left.225 \mathrm{nM}\right){ }^{3}$ Therefore, it is desirable to establish a highly sensitive and simple method to detect Tc.

As an important and effective analytical tool, fluorescence analysis has been widely used in the fields of life sciences, medicine and environmental monitoring. ${ }^{4-6}$ However, fluorescence quantum yields of some fluorescent materials are low, resulting in poor detection sensitivity, thus limiting their applications in fluorescence analysis. Plasmon-enhanced fluorescence (PEF) effect can greatly enhance fluorescence intensity of probes, leading to significantly increased sensitivity of detection, thus expanding the scope of applications. ${ }^{7,8}$

In recent years, studies on PEF materials mainly focused on metal nanoparticles, such as AgNPs and AuNPs. ${ }^{9,10}$ However, these materials have several shortcomings, such as poor light stability, low biocompatibility and relatively large optical loss. These shortcomings limit the application scope of PEF sensors. Therefore, it is necessary to explore novel materials that are

College of Materials Science and Engineering, Huaqiao University, Key Laboratory of Molecular Designing and Green Conversions (Fujian University), Xiamen 361021, China.E-mail: sunxy@hqu.edu.cn; Fax: +86-592-6160088; Tel: +86-592-6162231

$\uparrow$ Electronic supplementary information (ESI) available. See DOI: $10.1039 / \mathrm{c} 8 \mathrm{ra04581j}$ cheap, stable and biocompatible for use in surface enhanced fluorescence.

Graphene, a rising star in the world of materials, is widely used in various fields because of its outstanding electronic and optical properties. ${ }^{11}$ One of these properties is that under certain conditions, graphene can produce surface plasmon, similar to that of metal nanoparticles..$^{12}$ In addition, graphene surface plasmon has the following excellent properties: it has extremely high wave momentum; it is a strong longitudinal local plasmon; it has infrared to terahertz band work area; and it is highly adjustable compared to metal surface plasmon. ${ }^{13,14}$ It should be pointed out that there many reports have focused on enhanced Raman spectroscopy, but reports involving enhanced fluorescence spectroscopy are rare. ${ }^{15-17}$ Until now, only a few examples involving the study of the phenomenon of enhanced fluorescence by graphene have been reported, including studies on fluorescence enhancement of GeSi quantum dots, $\mathrm{SnO}_{2}$ nanostructures and $\mathrm{ZnO}$ nanostructures by graphene..$^{18-20}$

Self-assembled multilayers (SAMs) can be easily prepared and converted to devices, and therefore, SAMs have been widely applied in biochemical analysis. ${ }^{21,22}$ Our group has reported that solid-liquid interfacial detection based on fluorescent selfassembled multilayers can provide high sensitivity. ${ }^{23-25}$ SAMs can be employed to easily control the distance between graphene and fluorophores with a polyelectrolyte.

Recently, carbon dots (CDs) have attracted considerable attention due to easy preparation, high photochemical stability and good biocompatibility, compared with traditional semiconductor quantum dots and organic dyes..$^{26,27}$ Moreover, CDs have also been applied in the sensing of ions (such as $\mathrm{Hg}^{2+}$, $\mathrm{Cu}^{2+}$, and $\mathrm{ClO}^{-}$), ${ }^{28,29}$ temperature, ${ }^{30}$ biological molecules (for 
instance, dopamine, bilirubin, and $\mathrm{L}-\mathrm{D}$-arginine $)^{31-33}$ and proteins. ${ }^{34}$

In this study, we synthesized poly(diallyldimethylammonium) chloride functionalized graphene (PDDA-G) with different reduction degrees to study its fluorescence enhancement efficiency. CDs were selected as fluorescent probes. PDDA and sodium polystyrenesulfonate (PSS) polyelectrolyte assembling layers were used to control the distance between CDs and PDDAG surface (Scheme 1). Experimental results show that graphene can enhance fluorescence intensity of CDs-based SAMs. Efficiency of fluorescence enhancement of CDs was found to strongly depend on the distance between CDs and PDDA-G surface, graphene concentration, and degree of graphene reduction. This SAMs sensor could be further applied to detect trace Tc with limit of detection (LOD) of $0.9284 \mathrm{nM}$.

\section{Experimental}

\section{Chemicals and reagents}

Poly(diallyldimethylammonium)chloride (PDDA, MW 100 000$200000)$ and tetracycline hydrochloride (Tc) were purchased from Aladdin (Shanghai, China). Sodium polystyrenesulfonate (PSS, MW 70 000) was obtained from Acros (USA). Procaine hydrochloride, clindamycin hydrochloride, cefotaxime sodium, 1,3,5-triazine, and trimethoprim were supplied by Macklin (Shanghai, China). Graphene oxide (GO) was purchased from Nanjing Xianfeng Nano Co. Ltd (Nanjing, China). Other reagents were of analytical grade and used without further purification. Ultrapure water was prepared by a Milli-Q water purification system (USA). Carbon dots (CDs) were synthesized according to the reported literature. ${ }^{35}$

\section{Apparatus}

All fluorescence spectra were recorded on a HITACHI F-7000 fluorescence spectrometer (Hitachi, Japan). Absorption spectra were obtained from a UV-2600 UV-Vis spectrophotometer (Shimadzu, Japan) and a Cary 5000 UV-Vis-NIR spectrophotometer (Agilent, USA). Fluorescence images were obtained from a Leica TCS SP8 laser scanning confocal microscope (Leica, Germany). Fluorescence quantum yield measurements were conducted on a FLS-920 steady-state/transient fluorescence spectrometer (Edinburgh Instruments, UK). Fluorescence lifetime decay curves were obtained on a FS5 fluorescence spectrometer (Edinburgh Instruments, UK). Raman spectra were measured with an InVia confocal Raman spectrometer (Renishaw, UK). Thickness of the

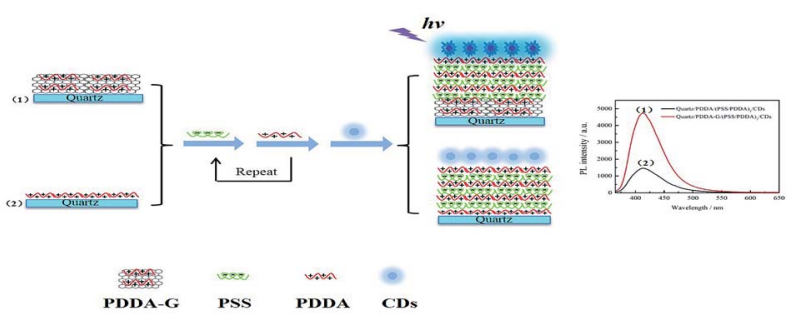

Scheme 1 Schematic representation of assembly of (1) quartz/PDDA$\mathrm{G} /(\mathrm{PSS} / \mathrm{PDDA})_{3} / \mathrm{CDs}$ and (2) quartz/PDDA/(PSS/PDDA) ${ }_{3} / \mathrm{CDs}$ SAMs. graphene film was recorded by atomic force microscopy (AFM, Mutimode-8J, America). The potentials of PDDA-G and CDs were recorded by a Zetasizer Nano ZS (Malvern Instruments Ltd., UK). Assembly process was monitored in real time using a quartz crystal microbalance (QCM, Q-Sense Biolin Scientific, Sweden). Morphology of SAMs was probed by using a scanning electron microscope (SEM, FEI Inspect F50, America). Morphology of CDs was recorded by using a transmission electron microscope (TEM, H-7650, Japan). PDDA-LG was synthesized using a Xe lamp (CHF$\mathrm{XM}-500 \mathrm{~W}$, China) as the light source.

\section{Construction of quartz/PDDA-G/(PSS/PDDA) $)_{n} /$ CDs SAMs}

Quartz substrates were hydroxylated as previously reported. ${ }^{23}$ Then, PDDA-G with different concentrations was poured onto the hydroxylated substrates and placed into an oven for $1 \mathrm{~h}$. Then, the substrates with PDDA-G were dipped into $1 \mathrm{~g} \mathrm{~L}^{-1}$ PSS and $1 \%(\mathrm{v} / \mathrm{v})$ PDDA $(0.1 \mathrm{M} \mathrm{NaCl})$, sequentially, for $1 \mathrm{~h}$. This process was repeated $n$ times. Finally, these substrates were immersed into a CDs solution for $3 \mathrm{~h}$ and quartz/PDDA-G/(PSS/ PDDA $)_{n} /$ CDs SAMs were obtained.

\section{Results and discussion}

\section{Characterization of PDDA-G}

The obtained PDDA-G was characterized by UV-Vis, Raman and FTIR. UV-Vis absorption spectra of GO and PDDA-G were recorded, as shown in Fig. 1a. Compared with GO, the $\pi-\pi^{*}$ transition of $\mathrm{C}=\mathrm{C}$ of PDDA-G shifts from $230 \mathrm{~nm}$ to $270 \mathrm{~nm}$ and the shoulder peak at $c a .300 \mathrm{~nm}$ disappears. Raman spectral results are presented in Fig. 1b. PDDA-G and GO show similar Raman spectra. The peak (D peak) at $c a .1350 \mathrm{~cm}^{-1}$ is the structural disorder peak and the graphite lattice defect peak, which is caused by vibration of $\mathrm{sp}^{3}$ hybrid carbon atoms. The $\mathrm{G}$ peak at $c a .1600 \mathrm{~cm}^{-1}$ is the typical Raman peak of bulk crystalline graphite, which is usually related to $\mathrm{sp}^{2}$ hybrid carbon atoms. In general, the degree of disorder of carbon material can be expressed as the ratio of the two integrated peak areas $\left(I_{\mathrm{D}} / I_{\mathrm{G}}\right)$. By fitting calculations, it was found that $I_{\mathrm{D}} / I_{\mathrm{G}}$ ratio of PDDA-G
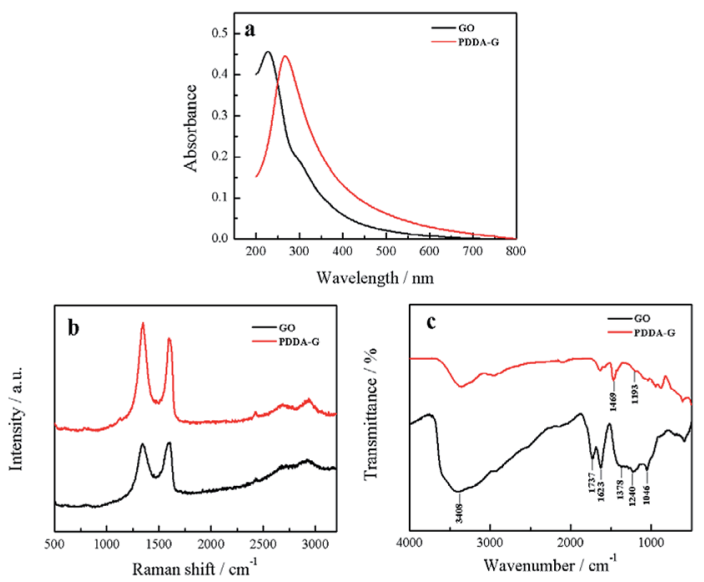

Fig. 1 (a) UV-Vis absorption spectra, (b) Raman spectra and (c) FTIR spectra of PDDA-G and GO. 
was 1.73 , which was higher than that of GO (1.35). This observation indicates that GO was reduced by hydrazine hydrate.

Furthermore, FTIR spectra were recorded to probe PDDA-G and the results are shown in Fig. 1c. GO shows a strong $-\mathrm{OH}$ stretching vibration absorption peak at $3408 \mathrm{~cm}^{-1}$ and the peak at $1378 \mathrm{~cm}^{-1}$ can be considered to originate from the deformation vibration of $-\mathrm{OH}$. The peaks at $1623 \mathrm{~cm}^{-1}$ and $1737 \mathrm{~cm}^{-1}$ correspond to the stretching vibration of $\mathrm{C}=\mathrm{O}$ in the carboxyl group. The peaks at $1240 \mathrm{~cm}^{-1}$ and $1046 \mathrm{~cm}^{-1}$ can be ascribed to the stretching vibrations of $\mathrm{C}=\mathrm{O}$ in the epoxy group and $\mathrm{C}-\mathrm{O}$ in the alkoxy group, respectively. However, the absorption peaks of these oxygen-containing functional groups disappeared after reduction with hydrazine hydrate, further supporting that GO was reduced to graphene. FTIR spectrum of PDDA-G show two new absorption peaks at $1469 \mathrm{~cm}^{-1}(\mathrm{C}-\mathrm{H})$ and $1193 \mathrm{~cm}^{-1}(\mathrm{C}-\mathrm{N})$, compared with the FTIR spectrum of GO, corresponding to the characteristic bands of PDDA. These observations suggest that graphene has been successfully functionalized by PDDA with a positive charge (Fig. S1a $\dagger$ ).

\section{Characterization of CDs}

Blue fluorescent CDs were prepared by hydrothermal treatment. TEM image of CDs reveals that the as-prepared CDs show spherical morphology and are uniform in overall distribution with an average particle size of $5.24 \mathrm{~nm}$ (Fig. 2). Zeta potential results show a value of $-23.02 \mathrm{mV}$, indicating that the CDs' surface has negative charges (Fig. S1a $\dagger$ ). FTIR spectra show the presence of amide functional groups on the surface of the CDs, in addition to hydroxyl, carboxyl and sulfhydryl groups (Fig. S2a $\dagger$ ). As shown in the UV-Vis absorption spectra (Fig. S3 $\dagger$ ), one absorption peak was located at $246 \mathrm{~nm}$, attributed to $\pi \rightarrow$ $\pi^{*}$ transition of $\mathrm{C}=\mathrm{C}$ bond, while another peak at $342 \mathrm{~nm}$ was attributed to $\mathrm{n} \rightarrow \pi^{*}$ transition of $\mathrm{C}=\mathrm{O}$ bond. Fluorescence spectra of CDs show that the maximum peak is centered at $421 \mathrm{~nm}$ under a $350 \mathrm{~nm}$ excitation wavelength.

\section{Characterization of SAMs}

In order to monitor the assembly process, QCM was employed to investigate the assembly of the layers. As the number of layers increased, the frequency continuously decreased and the corresponding dissipation continuously increased, indicating an increase in the mass of SAMs, as shown in Fig. 3. Therefore, it could be concluded that these layers had been deposited on the surface of the substrate by electrostatic interaction. The as-
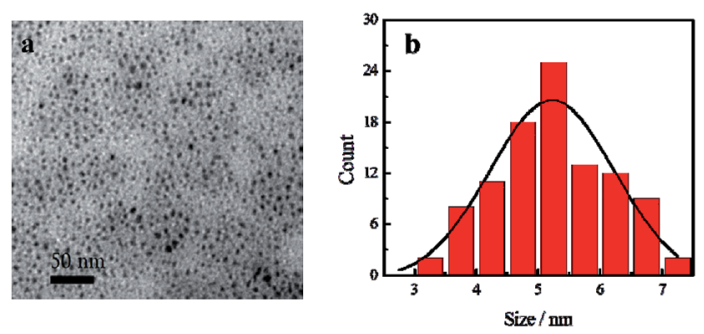

Fig. 2 (a) SEM image of CDs and (b) particle size distribution histogram of CDs.

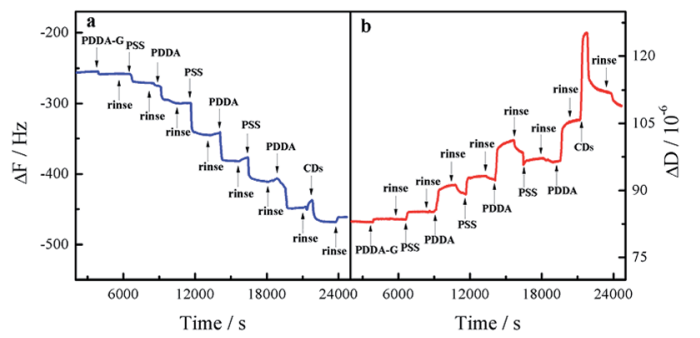

Fig. 3 Response in frequency (a) and dissipation (b) for fabrication process of quartz/PDDA-G/(PSS/PDDA) 3 /CDs SAMs.

prepared SAMs were characterized by SEM (Fig. S4†). We found that the entire surface of the SAMs was distributed uniformly with CDs. The as-prepared SAMs had good light stability, as shown in Fig. S5. $\dagger$

\section{Graphene fluorescence enhancement in SAMs}

In this study, PDDA-G and CDs were selected as the plasmatic material and the fluorophore, respectively. PDDA-G with positive charge can effectively avoid aggregation of graphene during the assembly process, which is beneficial for electrostatic assembly. CDs with negatively charged surface groups and excellent photostability were chosen as the fluorophore to probe fluorescence enhancement by graphene (Fig. $\mathrm{S} 1 \mathrm{~b} \dagger$ ).

As shown in Fig. 4, PDDA-G effectively enhances fluorescence intensity of CDs SAMs. The fluorescence intensity of CDS SAMs with graphene was $c a$. 3.2 times higher than that of CDs SAMs without graphene. As shown in Fig. S12, $\dagger$ we found that enhancement efficiency was closely related to excitation wavelength $^{18}$ and fluorescence enhancement efficiency was maximized at excitation wavelength of $350 \mathrm{~nm}$. Interestingly, the fluorescence lifetimes of CDs SAMs in the presence and absence of graphene were measured to be 4.342 ns and 5.721 ns, respectively. Corresponding quantum yields increased from $35.27 \%$ to $46.03 \%$. Considering reduced lifetimes and increased fluorescence quantum yields of SAMs with PDDA-G, the mechanism of graphene enhanced fluorescence should be similar to that of metal enhanced fluorescence.

The following experiments also confirmed that graphene could enhance fluorescence intensity of SAMs. The fluorescence microscopy images of quartz/PDDA-G/(PSS/PDDA) 3 /CDs SAMs and quartz/PDDA/(PSS/PDDA $)_{3} /$ CDs SAMs are shown in Fig. 5. It could be observed that fluorescence intensity of each region of

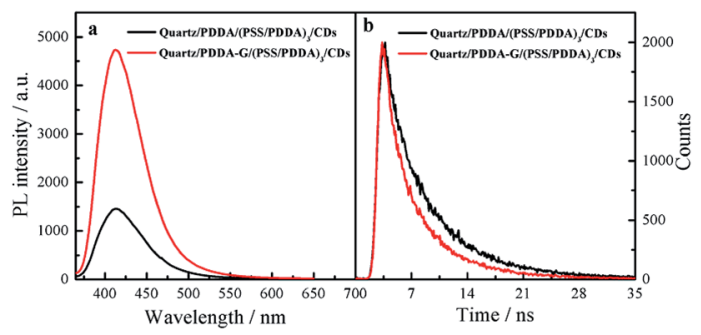

Fig. 4 Fluorescence spectra (a) and fluorescence decay curves (b) of quartz/PDDA/(PSS/PDDA) 3 /CDs SAMs and quartz/PDDA-G/(PSS/ $\mathrm{PDDA})_{3} / \mathrm{CDs}$ SAMs 

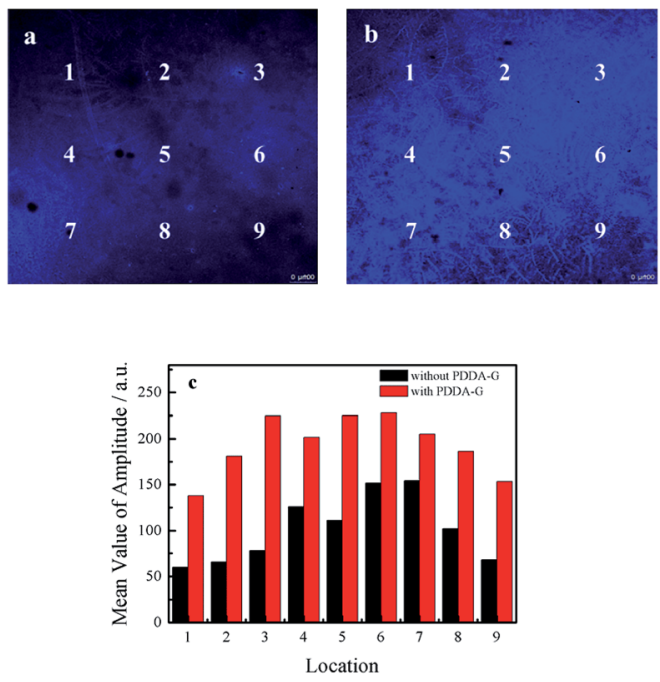

Fig. 5 Fluorescence microscopy images of (a) quartz/PDDA/(PSS/ PDDA) ${ }_{3} / C D s$ SAMs and (b) quartz/PDDA-G/(PSS/PDDA) $3 /$ CDs SAMs. (c) Confocal fluorescence values of quartz/PDDA/(PSS/PDDA) $3 / C D s$ SAMs and quartz/PDDA-G/(PSS/PDDA) 3 /CDs SAMs with different matching locations.

quartz/PDDA-G/(PSS/PDDA) $)_{3} /$ CDs SAMs was stronger than that of quartz/PDDA/(PSS/PDDA) $)_{3} / \mathrm{CDs}$ SAMs due to the presence of graphene in the former, as shown in Fig. 5c.

To further investigate the enhancing effect of graphene, UVVis absorption spectra of quartz/PDDA-G/(PSS/PDDA) $)_{3} /$ CDs SAMs and quartz/PDDA/(PSS/PDDA) $3 /$ CDs SAMs were recorded (Fig. S6 $\dagger$ ). Absorbance at $350 \mathrm{~nm}$ of CDs in these two SAMs was found to be unchanged, indicating that absorbed amounts of CDs on these two SAMs were consistent. Therefore, fluorescence intensity enhancement of CDs SAMs did not occur due to the increase in the amount of CDs in SAMs.

\section{Optimization of PDDA-G concentration}

To explore the effect of graphene concentration on fluorescence enhancement, PDDA-G assembled on quartz surface with concentrations of graphene varying from $0.001 \mathrm{mg} \mathrm{mL}^{-1}$ to $0.1 \mathrm{mg} \mathrm{mL} \mathrm{m}^{-1}$ was systematically studied. Results show that fluorescence intensity of CDs is PDDA-G concentrationdependent. Fluorescence intensity of CDs-based SAMs first increased and then decreased with the increase in PDDA-G concentration (Fig. S7†). When PDDA-G concentration was $0.005 \mathrm{mg} \mathrm{mL}^{-1}$, fluorescence intensity of CDs was enhanced and thickness of the graphene layer at this PDDA-G concentration was ca. $4 \mathrm{~nm}$ (Fig. S8†). Since the transmittance decreased with the increase in PDDA-G concentration, fluorescence intensity of CDs SAMs was quenched. ${ }^{36}$

\section{Optimization of layers of polyelectrolyte}

Fluorescence enhancement by graphene is dependent on the distance between CDs and PDDA-G surface. In this study, $(\mathrm{PDDA} / \mathrm{PSS})_{n}$ bilayers were employed to control the spacing distance between PDDA-G and CDs. The thickness of one bilayer of PSS/PDDA is $c a .3 \mathrm{~nm},{ }^{37}$ and the fluorescence spectra of CDs with different numbers of spacer layers on quartz/PDDA-

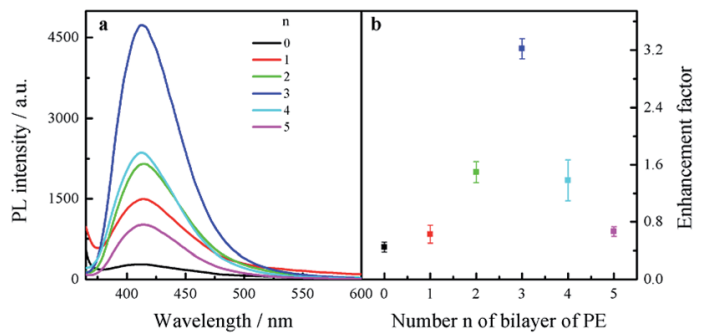

Fig. 6 (a) Fluorescence spectra of quartz/PDDA-G/(PSS/PDDA) ${ }_{n} / C D s$ SAMs with different layers of (PSS/PDDA $)_{n}(n=0-5)$. (b) Enhancement factor as a function of the number $n$ of PSS/PDDA bilayers.

G/(PSS/PDDA) ${ }_{n} /$ CDs SAMs were examined (Fig. 6a). To further understand the enhanced efficiency of graphene, the enhancement factor (EF) was defined as the following equation:

$$
\mathrm{EF}=\frac{F_{\mathrm{Gr}}}{F_{\mathrm{NGr}}}
$$

where $F_{\mathrm{Gr}}$ and $F_{\mathrm{NGr}}$. represent fluorescence intensity of SAMs with and without graphene, respectively.

Enhancement factor was plotted against the number of bilayers, as shown in Fig. 6b. The optimal layer number of polyelectrolytes was found to be 3 layers and thickness was $c a$. $9 \mathrm{~nm}$; corresponding EF was calculated to be ca. 3.2.

\section{Effect of degree of graphene reduction on fluorescence enhancement}

To investigate the effect of the change in graphene structure on fluorescence enhancement, graphene with different degrees of graphene reduction was synthesized under various reduction conditions, namely, room temperature, photochemical reduction and hydrothermal reaction. On conducting the synthesis reactions, the strongest peak in the UV-Vis absorption spectra of graphene was found to gradually shift from $230 \mathrm{~nm}$ to $270 \mathrm{~nm},{ }^{38}$ as shown in Fig. 7a, indicating that the degree of graphene reduction gradually increased. Moreover, Raman spectra of four different graphene samples were recorded. The increase in $I_{\mathrm{D}} / I_{\mathrm{G}}$ ratio indicated that the degree of graphitization gradually increased $^{39}$ (Fig. 7b). These experimental results showed that the degree of graphene reduction of PDDA-G was the highest. Degree of graphene reduction was found to have a significant effect on fluorescence enhancement efficiency (Fig. 8). As the degree of graphene reduction increased, the fluorescence enhancement efficiency continuously increased. However,

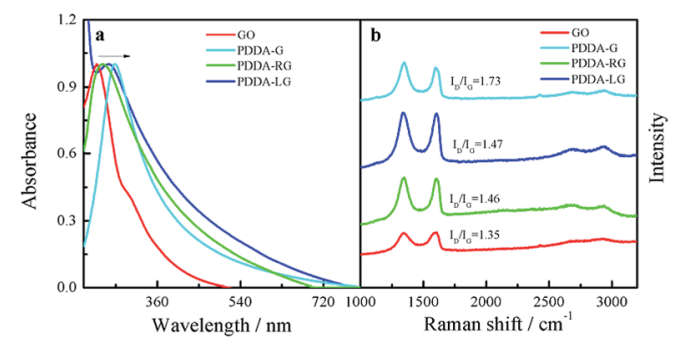

Fig. 7 (a) UV-Vis absorption spectra and (b) Raman spectra of GO, PDDA-G, PDDA-RG and PDDA-LG. 


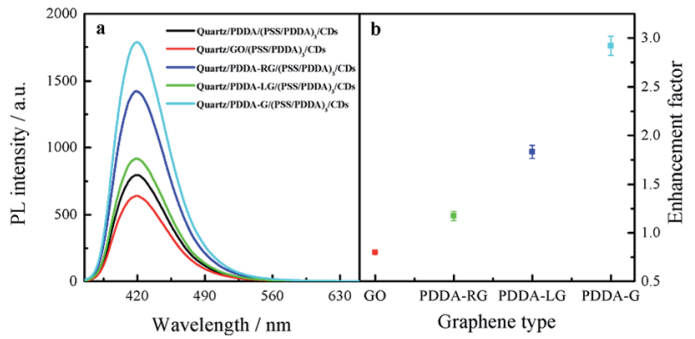

Fig. 8 (a) Fluorescence spectra of CDs SAMs and (b) enhancement factor of graphene with different degrees of graphene reduction.

fluorescence spectra of quartz/GO/(PSS/PDDA) $3 /$ CDs SAMs show much weaker intensity than those of other platforms, probably due to the enhanced energy transfer from CDs to GO. ${ }^{21}$

\section{Sensing for Tc based on inner filter effect}

Since graphene can enhance fluorescence of SAMs, detection sensitivity should be improved. Therefore, CDs-based SAMs with graphene were expected to develop a sensing device with high sensitivity. Our experiments revealed that fluorescence intensity of CDs could be effectively quenched by introduced Tc. In general, quenching mechanisms involve energy transfer, electron transfer, inner filter effects, etc. In this study, it was found that fluorescence lifetime of CDs did not change in the presence of Tc (Fig. S9b $\dagger$ ). To further probe the mechanism of fluorescence quenching by Tc, the reduction potential of CDs was detected as $-0.475 \mathrm{~V}$ (Fig. S10a $\dagger$ ). Then, the lowest unoccupied molecular orbital (LUMO) and highest occupied molecular orbital (HOMO) energy levels of CDs were calculated as $-3.93 \mathrm{eV}$ and $-7.47 \mathrm{eV}$, respectively. The $E_{\mathrm{LUMO}}$ and $E_{\mathrm{HOMO}}$ of Tc have been obtained as $-3.87 \mathrm{eV}$ and $-6.20 \mathrm{eV}$, respectively. ${ }^{40}$ Therefore, it was considered that electrons in the HUMO of CDs could not be transferred to the HUMO of Tc, as shown in Fig. S10b. $\uparrow$ Furthermore, emission spectra of CDs effectively overlap with absorption spectra of Tc (Fig. S9a †). Therefore, it can be deduced that CDs' fluorescence was quenched by Tc probably due to the inner filter effect (IFE) rather than energy transfer or electron transfer.

After optimization of experimental conditions, Tc at different concentrations was added into SAMs system. It was found that fluorescence intensity of SAMs in $10 \mathrm{mM}$ PB buffer solution of $\mathrm{pH} 6.8$ gradually decreased (Fig. 9a). The relationship between $F_{0} / F$ of quartz/PDDA-G/(PSS/PDDA) , $_{3}$ CDs SAMs and Tc concentration was established (Fig. 9b). It was found that when Tc concentration was varied in the range of 4.762$90.91 \mathrm{nM}$, a good linear relationship between the ratio $F_{0} / F$ and Tc concentration could be obtained as $F_{0} / F=6.432 \times 10^{6} \mathrm{C}+$ 1.003 , with $R^{2}=0.9924$, where $C$ is the Tc concentration with M as the unit and $F$ and $F_{0}$ represent the fluorescence intensity of SAMs in the presence or absence of Tc, respectively. LOD of CDs SAMs with graphene was measured and calculated to be $0.9284 \mathrm{nM}$, which is lower than $96 \mathrm{nM}$ of CDs SAMs without graphene and lower by 1-3 orders of magnitude than those of other reported methods (Table $\mathrm{S} 1 \dagger$ ).

The effect of antibacterial drugs and other related substances on quartz/PDDA-G/(PSS/PDDA) $)_{3} / \mathrm{CDs}$ SAMs were further studied. When Tc concentration was set to $56.60 \mathrm{nM}$,

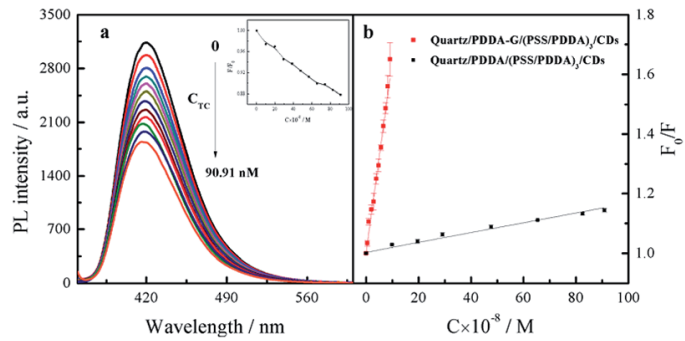

Fig. 9 (a) Fluorescence spectral response of quartz/PDDA-G/(PSS/ $\mathrm{PDDA})_{3} / \mathrm{CDs}$ SAMs toward Tc at different concentrations in $10 \mathrm{mM}$ PB buffer of $\mathrm{pH}$ 6.8. (Inset graph shows the ratio $F / F_{0}$ of fluorescence intensity varied with concentration of $\mathrm{Tc}$ ). (b) Relationship curves between $F_{0} / F$ of SAMs and Tc concentration.

Table 1 Detection of Tc in milk samples by this method

\begin{tabular}{lrrl}
\hline Spiked $(\mathrm{nM})$ & Detected $(\mathrm{nM})$ & Recovery $(\%)$ & $\operatorname{RSD}(n=3, \%)$ \\
\hline 9.96 & $9.99 \pm 0.32$ & 100.30 & 3.26 \\
29.13 & $29.06 \pm 1.33$ & 99.77 & 4.55 \\
65.42 & $66.32 \pm 1.26$ & 101.38 & 1.94
\end{tabular}

relative error was within the range of $\pm 10 \%$. Experimental results are listed in Table $\mathrm{S} 2 \dagger$ and indicate that SAMs have high selectivity for detecting Tc.

\section{Detection of Tc in milk}

The SAMs sensor was further applied for detecting Tc in milk samples. Milk samples were pre-processed according to reported literature. ${ }^{41}$ The milk samples were mixed with different concentrations of Tc. As shown in Table 1, recoveries of $99.77-101.38 \%$ and relative standard deviations of 1.94$4.55 \%$ were obtained, indicating that this method has favorable recovery and precision, suitable for application to practical analysis.

\section{Conclusions}

In summary, it was found that the fluorescence of SAMs could be greatly enhanced. The enhanced fluorescence efficiency was dependent on graphene concentration, degree of graphene reduction, and the distance between CDs and graphene surface. When the highly reduced graphene was used and CDs were chosen as fluorescence probe, fluorescence enhancement efficiency of SAMs was improved. Fluorescence intensity of CDsbased SAMs in the presence of graphene was increased by 3.2 times, with PSS/PDDA distance of 3 bilayers compared with CDS SAMs in the absence of graphene. The established quartz/PDDAG/(PSS/PDDA) $)_{3} /$ CDs SAMs were employed to detect trace Tc based on IFE between CDs and Tc. A good linear relationship between $F_{0} / F$ and Tc concentration was obtained. Compared with other reported detection techniques, this method showed excellent sensitivity with LOD of $0.9284 \mathrm{nM}$, which was 2 orders higher than that of SAMs in the absence of graphene. Therefore, graphene, which can be incorporated into a new generation of 
plasmatic materials for surface-enhanced fluorescence, is expected to be a substitute for traditional precious metal materials.

\section{Conflicts of interest}

There are no conflicts to declare.

\section{Acknowledgements}

This study was financially supported by the Natural Science Foundation of China (No. 21275059 and 21575044), the Natural Science Foundation of Fujian Province (No. 2015J01054 and 2016J01062), Foundation of Graphene Powder \& Composite Research Center of Fujian Province (2017H2001).

\section{References}

1 J. Li, L. Chen, X. Wang, H. Jin, L. Ding, K. Zhang and H. Zhang, Talanta, 2008, 75, 1245-1252.

2 F. Conzuelo, M. Gamella, S. Campuzano, A. J. Reviejo and J. M. Pingarron, Anal. Chim. Acta, 2012, 737, 29-36.

3 L. Q. Wang, F. Y. Lin and L. P. Yu, Analyst, 2012, 137, 35023509 .

4 J. Y. Hou, J. Dong, H. S. Zhu, X. Teng, S. Y. Ai and M. L. Mang, Biosens. Bioelectron., 2015, 68, 20-26.

5 S. A. A. Vandarkuzhali, V. Jeyalakshmi, G. Sivaraman, S. Singaravadivel, K. R. Krishnamurthy and B. Viswanathan, Sens. Actuators, B, 2017, 252, 894-900.

6 F. Samari, B. Hemmateenejad, Z. Rezaei and M. Shamsipur, Anal. Methods, 2012, 4, 4155-4160.

7 Z. Chen, H. Li, W. C. Jia, X. H. Liu, Z. M. Li, F. Wen, N. Zheng, J. D. Jiang and D. K. Xu, Anal. Chem., 2017, 89, 5901-5909.

8 N. Sui, K. Wang, L. N. Wang, F. X. Xie, T. L. Li, Q. Bai, D. Zhang, M. H. Liu and W. W. Yu, Sens. Actuators, B, 2017, 245, 568-573.

9 C. Vietz, I. Kaminska, M. Sanz Paz, P. Tinnefeld and G. P. Acuna, ACS Nano, 2017, 11, 4969-4975.

10 J. Jana, T. Aditya, M. Ganguly, S. K. Mehetor and T. Pal, Spectrochim. Acta, Part A, 2018, 188, 551-560.

11 J. W. Weber, V. E. Calado and M. C. M. van de Sanden, Appl. Phys. Lett., 2010, 97, 127403.

12 A. A. Dubinov, V. Y. Aleshkin, V. Ryzhii, M. S. Shur and T. Otsuji, J. Appl. Phys., 2014, 115, 044511.

13 E. H. Hwang and S. Das Sarma, Phys. Rev. B: Condens. Matter Mater. Phys., 2007, 75, 205418.

14 N. K. Emani, T. F. Chung, X. J. Ni, A. V. Kildishev, Y. P. Chen and A. Boltasseva, Nano Lett., 2012, 12, 5202-5206.

15 C. Srichan, M. Ekpanyapong, M. Horprathum, P. Eiamchai, N. Nuntawong, D. Phokharatkul, P. Danvirutai, E. Bohez, A. Wisitsoraat and A. Tuantranont, Sci. Rep., 2016, 6, 23733.

16 F. Yin, S. Wu, Y. Wang, L. Wu, P. Yuan and X. Wang, J. Solid State Chem., 2016, 237, 57-63.

17 S. M. Feng, M. C. dos Santos, B. R. Carvalho, R. T. Lv, Q. Li, K. Fujisawa, A. L. Elias, Y. Lei, N. Perea-Lopez, M. Endo, M. H. Pan, M. A. Pimenta and M. Terrones, Sci. Adv., 2016, 2, e1600322.
18 Y. L. Chen, Y. J. Ma, D. D. Chen, W. Q. Wang, K. Ding, Q. Wu, Y. L. Fan, X. J. Yang, Z. Y. Zhong, F. Xu and Z. M. Jiang, Appl. Phys. Lett., 2014, 105, 021104.

19 J. Ding, X. Yan, J. Li, B. Shen, J. Yang, J. Chen and Q. Xue, ACS Appl. Mater. Interfaces, 2011, 3, 4299-4305.

20 V. Khranovskyy, I. Shtepliuk, I. G. Ivanov, I. Tsiaoussis and R. Yakimova, Carbon, 2016, 99, 295-301.

21 X. Y. Sun, B. Liu, Y. F. Sun and Y. Yu, Biosens. Bioelectron., 2014, 61, 466-470.

22 X. Y. Sun, Y. Peng, Y. L. Lin, L. F. Cai, F. Li and B. Liu, Sens. Actuators, B, 2018, 255, 2121-2125.

23 B. Liu, X. P. Wang, X. Y. Sun, C. X. Yang and P. Li, Anal. Methods, 2017, 9, 282-286.

24 X. Sun, B. Liu, S. Li and F. Li, Spectrochim. Acta, Part A, 2016, 161, 33-38.

25 X. Sun, B. Liu, C. Yang and C. Li, Spectrochim. Acta, Part A, 2014, 131, 288-293.

26 S. Zhu, L. He, F. Zhang, M. Li, S. Jiao, Y. Li, M. Chen, X. E. Zhao and H. Wang, Talanta, 2016, 161, 769-774.

27 G. Li, H. Fu, X. Chen, P. Gong, G. Chen, L. Xia, H. Wang, J. You and Y. Wu, Anal. Chem., 2016, 88, 2720-2726.

28 R. Gui, H. Jin, Y. Wang and J. Sun, Sens. Actuators, B, 2017, 245, 386-394.

29 Z. Huang, F. Lin, M. Hu, C. Li, T. Xu, C. Chen and X. Guo, J. Lumin., 2014, 151, 100-105.

30 H. Yang, Y. Long, H. Li, S. Pan, H. Liu, J. Yang and X. Hu, J. Colloid Interface Sci., 2018, 516, 192-201.

31 W. He, R. Gui, H. Jin, B. Wang, X. Bu and Y. Fu, Talanta, 2018, 178, 109-115.

32 R. R. Anjana, J. S. A. Devi, M. Jayasree, R. S. Aparna, B. Aswathy, G. L. Praveen, G. M. Lekha and G. Sony, Microchim. Acta, 2018, 185, 11.

33 X. Q. Zeng, L. Zhang, J. D. Yang, Y. Guo, Y. M. Huang, H. Y. Yuan and Y. S. Xie, New J. Chem., 2017, 41, 1521615228.

34 R. Gui, W. He, H. Jin, J. Sun and Y. Wang, Sens. Actuators, B, 2018, 255, 1623-1630.

35 Y. Zhang and J. H. He, Phys. Chem. Chem. Phys., 2015, 17, 20154-20159.

36 S. W. Hwang, D. H. Shin, C. O. Kim, S. H. Hong, M. C. Kim, J. Kim, K. Y. Lim, S. Kim, S. H. Choi, K. J. Ahn, G. Kim, S. H. Sim and B. H. Hong, Phys. Rev. Lett., 2010, 105, 127403.

37 J. Geng, J. Liang, Y. Wang, G. G. Gurzadyan and B. Liu, J. Phys. Chem. B, 2011, 115, 3281-3288.

38 X. Zheng, Y. Peng and Y. Yang, J. Raman Spectrosc., 2017, 48, 97-103.

39 P. Solis-Fernandez, R. Rozada, J. I. Paredes, S. Villar-Rodil, M. J. Fernandez-Merino, L. Guardia, A. Martinez-Alonso and J. M. D. Tascon, J. Alloys Compd., 2012, 536, S532-S537.

40 M. Lin, H. Y. Zou, T. Yang, Z. X. Liu, H. Liu and C. Z. Huang, Nanoscale, 2016, 8, 2999-3007.

41 M. Ramezani, N. Mohammad Danesh, P. Lavaee, K. Abnous and S. Mohammad Taghdisi, Biosens. Bioelectron., 2015, 70, 181-187. 